PROCEEDINGS OF THE

AMERICAN MATHEMATICAL SOCIETY

Volume 140, Number 1, January 2012, Pages 109-117

S 0002-9939(2011)11021-9

Article electronically published on May 20, 2011

\title{
BOUNDING THE FIRST HILBERT COEFFICIENT
}

\author{
KRISHNA HANUMANTHU AND CRAIG HUNEKE
}

(Communicated by Irena Peeva)

\begin{abstract}
This paper gives new bounds on the first Hilbert coefficient of an ideal of finite colength in a Cohen-Macaulay local ring. The bound given is quadratic in the multiplicity of the ideal. We compare our bound to previously known bounds and give examples to show that at least in some cases it is sharp. The techniques come largely from work of Elias, Rossi, Valla, and Vasconcelos.
\end{abstract}

\section{INTRODUCTION}

Throughout this paper we study a commutative Noetherian Cohen-Macaulay local ring $R$ with maximal ideal $\mathfrak{m}$ and infinite residue field $k$. Let $I$ be an $\mathfrak{m}$ primary ideal. We let $d$ be the dimension of $R$, we use $\mu(\quad)$ to denote the minimal number of generators, and use $\lambda(\quad)$ to denote the length of an $R$-module. We can write

$$
\lambda\left(R / I^{n+1}\right)=e_{0}(I)\left(\begin{array}{c}
n+d \\
d
\end{array}\right)-e_{1}(I)\left(\begin{array}{c}
n+d-1 \\
d-1
\end{array}\right)+\cdots+(-1)^{d} e_{d}(I),
$$

where the $e_{j}(I)$ are integers called the Hilbert coefficients (of $I$ ), and the equation is valid for all large values of $n$. There are many studies of relationships between the various Hilbert coefficients and bounds for them. Such bounds are necessary, for example, to prove finiteness of Hilbert functions of ideals with fixed multiplicity. The work of Srinivas and Trivedi [ST] does exactly this. Northcott [N] gave one of the first such bounds. He proved that if $R$ and $I$ are as above, then $e_{1}(I) \geq$ $e_{0}(I)-\lambda(R / I)$. This bound can be improved by adding another term involving the reduction number of $I$. An ideal $J \subset I$ is a reduction of $I$ if $I^{n+1}=J I^{n}$ for all large $n$. Let $J$ be a reduction of $I$. The reduction number of $I$ with respect to $J$, denoted $r_{J}(I)$, is the least integer $r$ such that $I^{r+1}=J I^{r}$. The least reduction number of $I$ with respect to all reductions is called the reduction number of $I$, denoted $r(I)$. Rossi $\left[\mathrm{Ro}\right.$ proved that if $d \leq 2$, then $e_{1}(I) \geq e_{0}(I)-\lambda(R / I)+r(I)-1$.

In this paper we give an upper bound on $e_{1}(I)$, as opposed to the lower bound given by Northcott. Several upper bounds exist in the literature. In RV1, Proposition 2.10], the bound $e_{1}(I) \leq\left(\begin{array}{c}e_{0}(I)-k+1 \\ 2\end{array}\right)$ is given for filtrations; in our context, their assumption is that $I \subset \mathfrak{m}^{k}$. Elias [E1, Proposition 2.5] generalized this bound

Received by the editors November 10, 2010

2010 Mathematics Subject Classification. Primary 13A30, 13B22, 13D40, 13H15.

The first author was partially supported by Robert D. Adams Visiting Assistant Professorship Fund.

The second author was partially supported by the National Science Foundation, grant DMS0756853 .

(C)2011 American Mathematical Society Reverts to public domain 28 years from publication 
by proving that $e_{1}(I) \leq\left(\begin{array}{c}e_{0}(I)-k \\ 2\end{array}\right)$ if $I \subset \mathfrak{m}^{k}$ and the integral closure of $I$ is not the integral closure of $\mathfrak{m}^{k}$. Our main result gives another bound in dimension one which can then be extended to higher dimensions and which in general is stronger than the previously known bounds. As a corollary, our main result can be used to recover, and slightly extend, the result of Elias stated above. He also gives other bounds, which we compare to ours in Section 3. Our methods come from those in RVV], but with one additional twist. We also show that our results are sharp by giving a sequence of examples.

\section{Dimension One}

It is well-known that $e_{1}(I)$ and $e_{0}(I)$ can be preserved after going modulo general elements of $I$ until one reaches dimension one. Thus the one-dimensional case is crucial. But one must also possibly preserve other assumptions, and this causes some difficulties in obtaining optimal results. Our first theorem handles the case of dimension one.

Theorem 2.1. Let $(R, \mathfrak{m})$ be a Cohen-Macaulay local ring of dimension one, and let $I \subset R$ be an $\mathfrak{m}$-primary ideal. Suppose that there exist distinct integrally closed ideals $J_{1}, \cdots, J_{k-1}$ such that $\mathfrak{m} \supsetneq J_{k-1} \supseteq J_{k-2} \supseteq \cdots \supseteq J_{1} \supsetneq \bar{I}$, where $\bar{I}$ is the integral closure of $I$. Then $e_{1}(I) \leq\left(\begin{array}{c}e_{0}(I)-k \\ 2\end{array}\right)$. If $I$ is not integrally closed, $e_{1}(I)<\left(\begin{array}{c}e_{0}(I)-k \\ 2\end{array}\right)$.

Proof. For simplicity we write $e_{j}(I)=e_{j}$.

We have $\lambda(R / \mathfrak{m})=1, \lambda\left(\mathfrak{m} / J_{k-1}\right) \geq 1, \lambda\left(J_{i+1} / J_{i}\right) \geq 1$ for $i=1, \ldots, k-2$, and finally $\lambda\left(J_{1} / I\right) \geq 1$. Thus $\lambda(R / I) \geq k+1$.

Let $r$ be the reduction number of $I$.

By the Eakin-Sathaye theorem [ES, we see that for $n \leq r, I^{n}$ cannot be generated by fewer than $n+1$ elements. So $\lambda\left(I^{n} / \mathfrak{m} I^{n}\right) \geq n+1$. Consider $\lambda\left(\mathfrak{m} I^{n} / I^{n+1}\right)$. We claim this length is at least $k$. To see this, consider the chain of ideals,

$$
\mathfrak{m} I^{n} \supset J_{k-1} I^{n} \supset J_{k-2} I^{n} \supset \ldots \supset J_{1} I^{n} \supset \bar{I} I^{n} .
$$

None of the ideals in this chain are equal; if for example $J_{l+1} I^{n}=J_{l} I^{n}$, then the determinantal trick (see $[\underline{\mathrm{SH}}, 1.1 .8]$ ) shows that $J_{l+1}$ is integral over $J_{l}$, a contradiction. Combining the above two inequalities, we obtain that $\lambda\left(I^{n} / I^{n+1}\right) \geq n+k+1$ for $n \leq r$.

We have $e_{0} n-e_{1}=\lambda\left(R / I^{n}\right)$ for $n \gg 0$. In fact, this equality holds for $n=r+1$ and $e_{1}=(r+1) e_{0}-\lambda\left(R / I^{r+1}\right)$. We rewrite this equality in the following way:

$$
e_{1}=\left(e_{0}-\lambda(R / I)\right)+\sum_{n=1}^{r}\left(e_{0}-\lambda\left(I^{n} / I^{n+1}\right)\right) .
$$

Using the inequalities above, we obtain that

$$
e_{1} \leq e_{0}-(k+1)+r e_{0}-\sum_{n=1}^{r}(n+k+1) .
$$

We claim that

$$
e_{0}-(k+1)+r e_{0}-\sum_{n=1}^{r}(n+k+1) \leq\left(\begin{array}{c}
e_{0}-k \\
2
\end{array}\right),
$$

which will finish the proof. 
To simplify this, we set $\alpha=\frac{r}{e_{0}}$. Note that

$$
\sum_{n=1}^{r}(n+k+1)=\frac{(r+k+1)(r+k+2)}{2}-\frac{(k+1)(k+2)}{2} .
$$

Now a simple calculation shows that

$$
\begin{aligned}
& \left(\begin{array}{c}
e_{0}-k \\
2
\end{array}\right)-\left(e_{0}-(k+1)+r e_{0}-\sum_{n=1}^{r}(n+k+1)\right) \\
= & \left(e_{0}(1-\alpha)\right)^{2}-e_{0}(1-\alpha)(2 k+3)+(k+1)(k+2) .
\end{aligned}
$$

Setting $x=e_{0}(1-\alpha)$, our claim becomes

$$
x^{2}-(2 k+3) x+(k+1)(k+2)=(x-(k+1))(x-(k+2)) \geq 0 .
$$

This is clear since $x=e_{0}-r$ is an integer and both terms are therefore both positive or both negative.

We let $K$ be an infinite field for the following examples.

Example 2.2. Let $R=K[[x, y]] /\left(x y^{2}\right)$ and let $I=\left(x^{2}, y\right) . R$ is a Cohen-Macaulay local ring of dimension 1 and $I$ is $\mathfrak{m}$-primary. Moreover, $I$ is an integrally closed ideal and $I \neq \mathfrak{m}$. So we can apply Theorem 2.1 with $k=1$.

We have that $I^{n}=\left(x^{2 n}, x^{2 n-2} y, y^{n}\right)$. Since $x y^{2}=0$ in $R$, a product of $n$ elements of $\left\{x^{2}, y\right\}$ is zero if $y$ is taken at least twice and $x^{2}$ is taken at least once.

A $K$-basis of $R / I^{n}$ is $\left\{1, x, \ldots, x^{2 n-1}, y, \ldots, y^{n-1}, x y, \ldots, x^{2 n-3} y\right\}$.

Hence $\lambda\left(R / I^{n}\right)=5 n-4$, which implies that $e_{0}(I)=5$ and $e_{1}(I)=4$. So the conclusion of Theorem 2.1 holds. Notice also that $e_{1}(I) \nless\left(\begin{array}{c}e_{0}(I)-2 \\ 2\end{array}\right)$.

Example 2.3. $R=K[[x, y]] /(x y(x-y))$ and $I_{k}=\left(x^{k+1}, y\right), k \geq 1$.

We claim that the reduction number of $I$ is 1 . It suffices to prove that $I^{2}=z I$ with $z=y+x^{k+1}$. To prove this we can lift back to $K[[x, y]]$ and prove that $\left(x^{k+1}, y\right)^{2} \subset\left(z y, z x^{k+1}, x^{2} y-y x^{2}\right)$. In fact it is clear that it suffices to see that $y^{2} \in\left(z y, z x^{k+1}, x^{2} y-y x^{2}\right)$. But $y^{2}\left(1+x^{k}\right)=z y-x^{k-1}\left(x^{2} y-x y^{2}\right)$. As $1+x^{k}$ is a unit, our claim is proved. It is well-known that having reduction number equal to 1 implies that $e_{0}(I)=\lambda\left(I / I^{2}\right)$. This latter can be easily taken to be $k+3$. Further, $\lambda\left(R / I^{2}\right)=2 e_{0}(I)-e_{1}(I)$. A simple calculation shows that $\lambda\left(R / I^{2}\right)=2 k+4$. So $e_{1}(I)=2$. Observe that the ideals $I_{k}$ are integrally closed for every $k$. This can be seen, e.g., by going modulo $y$. Therefore we have a chain of distinct integrally closed ideals,

$$
\mathfrak{m} \supset I_{1} \supset I_{2} \supset \ldots \supset I_{k+1} \text {. }
$$

Applying Theorem 2.1 yields $e_{1}(I)<\left(\begin{array}{c}e_{0}(I)-k \\ 2\end{array}\right)$, but $e_{1}(I) \nless\left(\begin{array}{c}e_{0}(I)-k-1 \\ 2\end{array}\right)$.

In these examples, $e_{1}(I)$ has the smallest possible value, namely $e_{0}(I)-\lambda(R / I)$.

Example 2.4. $R=K[[x, y]] /\left(x^{2} y^{2}\right)$ and let $I=\left(x^{2}, y\right)$. Then $e_{o}(I)=e_{1}(I)=6$ and $\lambda(R / I)=2$. Here we may apply Theorem 2.1 with $k=1$. However, it is true also that $e_{1}(I) \leq\left(\begin{array}{c}e_{0}(I)-2 \\ 2\end{array}\right)$.

Theorem 2.1 shows that the maximum length of a chain of integrally closed ideals in $R$ which contain a given $\mathfrak{m}$-primary ideal $I$ is important to understand. The next result gives a good lower bound on this number which depends on the number of maximal ideals in the integral closure of $R$. We apply this in higher dimensions in Theorem 3.4 . 
Theorem 2.5. Let $(R, \mathfrak{m}, k)$ be a one-dimensional analytically unramified local domain with infinite residue field $k$. Set $S$ equal to the integral closure of $R$. Then $S$ is a semilocal domain; set $t=\operatorname{dim}_{k}(S / J a c(S))$, where $J a c(S)$ is the Jacobson radical of $S$. Let $I$ be an integrally closed ideal of $R$. Then there exists a chain of distinct integrally closed ideals, $\mathfrak{m} \supset J_{n-1} \supset \ldots \supset J_{0}=I$, where $n=\left\lfloor\frac{\lambda(R / I)-1}{t}\right\rfloor$.

Proof. Since $R$ is analytically unramified, $S$ is a module-finite extension of $R \underline{\underline{S H}}$, Theorem 9.2.2]. Thus $S$ is a Noetherian semi-local one-dimensional integrally closed domain and is therefore a PID. Since $k$ is infinite, we may choose a minimal reduction $x$ of $I$. In this case $x S=I S$ (note that every ideal of $S$ is integrally closed, and $I S$ is certainly in the integral closure of $x S$, and is thus equal to $x S)$.

Choose $J \neq I$ to be an integrally closed ideal containing $I$ such that $\lambda(J / I)$ is minimal. To prove the theorem, it suffices to prove that $\lambda(J / I) \leq t$. To see this, set $J_{1}=J$, and define $J_{i}$ inductively by repeating this step by replacing $I$ by $J_{i-1}$. Note that $\lambda\left(J_{i} / I\right) \leq i t$. Provided $J_{i} \neq \mathfrak{m}$ (i.e., it $\left.<\lambda(R / I)-1\right)$, we can continue this chain. It follows that a chain $\mathfrak{m} \supset J_{n-1} \supset \ldots \supset J_{0}=I$ exists if $n \leq \frac{\lambda(R / I)-1}{t}$.

Let $y \in R$ be a minimal reduction of $J$. We have then that $y S=J S$. By way of a contradiction, suppose that $\lambda(J / I) \geq t+1$, and choose elements $y, z_{1}, \ldots, z_{t} \in J$ such that $I \subset(I, y) \subset\left(I, y, z_{1}\right)=I_{1} \subset \ldots \subset\left(I, y, z_{1}, \ldots, z_{t}\right)=I_{t}$ is a chain of distinct ideals. Since all the elements $z_{j}$ are in the integral closure of $(I, y)$, it follows that $y S=I_{1} S=\ldots=I_{t} S$. Write $z_{j}=y s_{j}$ for some $s_{j} \in S, 1 \leq j \leq t$. Let $\mathfrak{n}_{1}, \ldots, \mathfrak{n}_{l}$ be the maximal ideals of $S$, so that $\operatorname{Jac}(S)=\mathfrak{n}_{1} \cap \ldots \cap \mathfrak{n}_{l}$. By assumption, $T=S /\left(\mathfrak{n}_{1} \cap \ldots \cap \mathfrak{n}_{l}\right) \cong k^{t}$. Let $\alpha_{j}$ be the image of $s_{j}$ in $T$. As $T$ is a $t$-dimensional vector space over $k$, there is a $k$-linear relation $\beta+\gamma_{1} \alpha_{1}+\ldots+\gamma_{t} \alpha_{t}=0$, where not all of $\beta, \gamma_{1}, \ldots, \gamma_{t}$ are zero.

Choose $r, r_{1}, \ldots, r_{t} \in R$ such that $r_{i} \equiv \gamma_{i}$ modulo $\mathfrak{m}$, and $r \equiv \beta$ modulo $\mathfrak{m}$. If any $\gamma_{i}$ or $\beta$ is 0 , we choose the corresponding lift to be 0 as well. Note that not all of $r, r_{1}, \ldots, r_{t}$ are in $\mathfrak{m}$, so at least one is a unit. By multiplying by $y$, we obtain that $u=r y+r_{1} z_{1}+\ldots+r_{t} z_{t} \in\left(\mathfrak{n}_{1} \cap \cdots \cap \mathfrak{n}_{l}\right) y \cap R$. Therefore $u S \subsetneq y S$.

We claim that $(I, u) S \subsetneq y S$ as well. Clearly $(I, u) S$ is contained in $y S$. To see they are not equal it suffices to prove they are not equal in some localization $S_{\mathfrak{n}_{i}}$ for some $1 \leq i \leq l$. But since $u \in\left(\mathfrak{n}_{1} \cap \cdots \cap \mathfrak{n}_{l}\right) y$, we get equality in every localization if and only if $I S_{\mathfrak{n}_{i}}=y S_{\mathfrak{n}_{i}}$ for all $i$. This forces $I S=y S$, a contradiction. Hence $(I, u) S \subsetneq y S$. This shows that the integral closure of $(I, u)$ is strictly inside $J$.

We also claim that $u \notin I$. This then contradicts the choice of $J$. We may write $u=r y+r_{1} z_{1}+\ldots+r_{j} z_{j}$, where $r_{j} \neq 0$. By the choice of liftings, it follows that $r_{j} \notin \mathfrak{m}$ and is therefore a unit. If $u \in I$, we obtain that $z_{j} \in\left(I, y, z_{1}, \ldots, z_{j-1}\right)$, a contradiction.

Definition 2.6. Let $(R, \mathfrak{m}, k)$ be a one-dimensional analytically unramified local domain with infinite residue field $k$. Set $S$ equal to the integral closure of $R$. We define the essential rank of $R$ to be $t=\operatorname{dim}_{k}(S / \operatorname{Jac}(S))$, where $\operatorname{Jac}(S)$ is the Jacobson radical of $S$.

We think of the essential rank as the possible number of maximal ideals of $S$, even after some finite extension.

We will now use the chain constructed in the above theorem to bound the first Hilbert coefficient.

Corollary 2.7. Let $(R, \mathfrak{m}, k)$ be a one-dimensional analytically unramified CohenMacaulay local domain with infinite residue field $k$. Let I be an $\mathfrak{m}$-primary ideal of 
$R$ with integral closure $\bar{I}$. Let $t$ be the essential rank of $R$. Then $e_{1}(I) \leq\left(\begin{array}{c}e_{0}(I)-n \\ 2\end{array}\right)$, where $n=\left\lfloor\frac{\lambda(R / \bar{I})-1}{t}\right\rfloor$.

Proof. By Theorem 2.5, we have a chain of distinct integrally closed ideals, $\mathfrak{m} \supset$ $J_{n-1} \supset \ldots \supset J_{0}=\bar{I}$, where $n=\left\lfloor\frac{\lambda(R / \bar{I})-1}{t}\right\rfloor$. Moreover, $e_{0}(I)=e_{0}(\bar{I}) \underline{\mathrm{SH}}$, Theorem 11.3.1]. It is then easy to see that $e_{1}(I) \leq e_{1}(\bar{I})$. The conclusion now follows from Theorem 2.1

Corollary 2.8. Let $(R, \mathfrak{m}, k)$ be a one-dimensional analytically unramified CohenMacaulay local domain with algebraically closed residue field $k$. Let $I$ be an $\mathfrak{m}-$ primary ideal of $R$ with integral closure $\bar{I}$. Let $t$ be the number of distinct maximal ideals of the integral closure of $R$. Then $e_{1}(I) \leq\left(\begin{array}{c}e_{0}(I)-n \\ 2\end{array}\right)$, where $n=\left\lfloor\frac{\lambda(R / \bar{I})-1}{t}\right\rfloor$.

Proof. This follows immediately from Corollary 2.7 by observing that since $k$ is algebraically closed, the essential rank of $R$ is exactly $t$.

Corollary 2.9. Let $(R, \mathfrak{m}, k)$ be a one-dimensional analytically irreducible CohenMacaulay local domain with algebraically closed residue field $k$. Let $I$ be an $\mathfrak{m}-$ primary ideal of $R$ with integral closure $\bar{I}$. Then $e_{1}(I) \leq\left(\begin{array}{c}e_{0}(I)-\lambda(R / \bar{I})+1 \\ 2\end{array}\right)$.

Proof. The completion $\hat{R}$ of $R$ (with respect to $\mathfrak{m}$ ) is a domain, and hence the number of minimal prime ideals of $\hat{R}$ is 1 . This shows that the number of maximal ideals of the integral closure of $R$ is 1 [SH, Proposition 4.3.2]. The desired inequality follows from Corollary 2.8 .

\section{Higher DIMENSION}

In order to extend the above results on bounding the first Hilbert coefficient to higher dimensions, we use a construction to reduce to the one-dimensional case. We set up this construction below.

Let $(R, \mathfrak{m}, k)$ be an analytically unramified Cohen-Macaulay local domain with an infinite residue field $k$. Let $I$ be an $\mathfrak{m}$-primary ideal of $R$, with integral closure $\bar{I}$. Let $d$ be the dimension of $R$. Choose a minimal reduction $y, x_{2}, \ldots, x_{d}$ of $I$. This sequence is a regular sequence since $R$ is Cohen-Macaulay. Set $T=R\left[\frac{x_{2}}{y}, \ldots, \frac{x_{d}}{y}\right]$. By [SH, Corollary 5.5.9], $T \cong R\left[T_{2}, \ldots, T_{d}\right] /\left(y T_{2}-x_{2}, \ldots, y T_{d}-x_{d}\right)$. It follows that the extension of $\mathfrak{m}$ to $T$ is a height one prime ideal; set $A=T_{\mathfrak{m} T}$. Observe that $A$ is a one-dimensional analytically unramified domain with an infinite residue field. We let $S$ be the integral closure of $T$. Note that $S$ is also the integral closure of $R\left[\frac{I}{y}\right]$. Set $B=W^{-1} S$, where $W=T \backslash \mathfrak{m} T$. Clearly $B$ is the integral closure of $A$.

Lemma 3.1. Let $(R, \mathfrak{m}, k)$ be an analytically unramified Cohen-Macaulay local domain and assume that the dimension of $R$ is at least 2 . Let $I$ be an integrally closed $\mathfrak{m}$-primary ideal of $R$ with a minimal reduction $\left(y, x_{2}, \ldots, x_{d}\right)$. Let $T \cong R\left[T_{2}, \ldots, T_{d}\right] /\left(y T_{2}-x_{2}, \ldots, y T_{d}-x_{d}\right)$ and $A=T_{\mathfrak{m} T}$ be as in the above construction. Set $J=I A$. Then $J$ is integrally closed, $e_{0}(I)=e_{0}(J), e_{1}(I)=e_{1}(J)$ and $\lambda(R / I)=\lambda(A / J)$.

Proof. We may assume that $y, x_{2}, \ldots, x_{d}$ is a superficial sequence for $I$. The fact that $e_{0}(I)=e_{0}(J)$ is proved in $\left[\mathrm{K}\right.$, Theorem 1.1]. We note that $e_{0}(I)=$ $e_{0}\left(\left(y, x_{2}, \ldots, x_{d}\right)\right)$. It also follows from the proof of Theorem 1.1 in $[\mathrm{K}]$, as on page 1022 , that the sequence $y T_{2}-x_{2}, \ldots, y T_{d}-x_{d} \in R\left[T_{2}, \ldots, T_{d}\right]_{\mathfrak{m} R\left[T_{2}, \ldots, T_{d}\right]}$ is a superficial sequence of length $d-1$ for $I R\left[T_{2}, \ldots, T_{d}\right]_{\mathfrak{m} R\left[T_{2}, \ldots, T_{d}\right]}$. It is well-known 
that modding out by a superficial sequence of length $d-1$ preserves both $e_{0}$ and $e_{1}$; for example, see [Ro, p. 1330].

To see that $J$ is integrally closed, let $C=R\left[\frac{x_{2}}{y}\right]_{\mathfrak{m}\left[\frac{x_{2}}{y}\right]}$. It follows from [I, Lemma 7] that $\overline{\left(y, x_{2}, \ldots, x_{d}\right) C}=\overline{\left(y, x_{2}, \ldots, x_{d}\right)} C$. Since $\overline{\left(y, x_{2}, \ldots, x_{d}\right)}=I$, we conclude that $I C=\overline{I C}$. Thus $I C$ is integrally closed. Clearly $\operatorname{dim} C=d-1$ and $y, x_{3}, \ldots, x_{d}$ is a system of parameters of $C$. Moreover, they form a regular sequence. Hence $C$ is Cohen-Macaulay. It is also clear that $C$ is analytically unramified. If $d-1 \geq 2$, we may apply [I, Lemma 7] to $C$ and the ideal $\left(y, x_{3}, \ldots, x_{d}\right) C$ and conclude as above that $I R\left[\frac{x_{1}}{y}, \frac{x_{2}}{y}\right]_{\mathfrak{m}\left[\frac{x_{1}}{y}, \frac{x_{2}}{y}\right]}$ is integrally closed. Proceeding this way, we obtain that $J$ is integrally closed.

Let $I^{\prime}=\left(y, x_{2}, \ldots, x_{d}\right) \subset R . \quad I^{\prime} T=y T$. It remains to prove that $\lambda(R / I)=$ $\lambda(A / J)$. However, by [SH, Prop. 8.4.2(6)], it follows that $\lambda(R / I)=\lambda(B / I B)$, where $B=R\left[T_{2}, \ldots, T_{d}\right]_{\mathfrak{m} R\left[T_{2}, \ldots, T_{d}\right]}$. Since the ideal $\left(y T_{2}-x_{2}, \ldots, y T_{d}-x_{d}\right) B$ is contained in $I B$, it follows that $\lambda(B / I B)=\lambda(A / J)$.

Definition 3.2. Let $(R, \mathfrak{m}, k)$ be an analytically unramified Cohen-Macaulay local domain with an infinite residue field $k$. Let $y, x_{1}, \ldots, x_{d}$ be a regular sequence in $R$. We define the essential rank of $\left(y, x_{2}, \ldots, x_{d}\right)$ to be the essential rank of the onedimensional ring $A$ constructed above. Let $I$ be an $\mathfrak{m}$-primary ideal of $R$. Define the essential rank of $I$ to be the minimum of essential ranks of minimal reductions of $I$.

Discussion 3.3. It is well-known that the number of maximal ideals in the integral closure of the ring $A$ in the above definition is exactly the number of Rees valuations of the ideal $I$. Suppose that $\mathfrak{n}$ is one of these maximal ideals in the integral closure $B$ of $A$. Then $B_{\mathfrak{n}}$ is a DVR. If for all such $\mathfrak{n}$ the residue field $B / \mathfrak{n}$ is purely transcendental over the residue field $k$ of $R$, then the essential rank of $I$ is exactly the number of Rees valuations of $I$. However, this property of being purely transcendental will rarely happen. If $I$ is a simple integrally closed ideal in a two-dimensional regular local ring, then it has exactly one Rees valuation, and the residue field of the valuation is purely transcendental over the residue field of $R$, provided this latter residue field is algebraically closed. See $[\mathrm{H}$, Cor. 4.4] for details. Dale Cutkosky has pointed out to us that at least in dimension three or higher, or in dimension two for nonrational singularities, the residue fields of the Rees valuations are seldom purely transcendental over the residue field of the base ring. The essential rank is closely related to the degree function introduced by David Rees [Re].

Theorem 3.4. Let $(R, \mathfrak{m}, k)$ be an analytically unramified Cohen-Macaulay local domain with infinite residue field $k$. Let $I$ be an $\mathfrak{m}$-primary ideal of $R$, with integral closure $\bar{I}$. Let $t$ denote the essential rank of $I$. Then $e_{1}(I) \leq\left(\begin{array}{c}e_{0}(I)-n \\ 2\end{array}\right)$, where $n=\left\lfloor\frac{\lambda(R / \bar{I})-1}{t}\right\rfloor$.

Proof. If $d=\operatorname{dim}(R)=1$, then the essential rank of $I$ is the same as the essential rank of $R$. So the result follows from Corollary 2.7. Assume that $d \geq 2$.

Choose a minimal reduction $y, x_{2}, \ldots, x_{d}$ of $I$ such that essential rank of $I$ is equal to the essential rank of $\left(y, x_{2}, \ldots, x_{d}\right)$. Let $T=R\left[\frac{x_{2}}{y}, \ldots, \frac{x_{d}}{y}\right] \cong R\left[T_{2}, \ldots, T_{d}\right] /\left(y T_{2}-\right.$ $\left.x_{2}, \ldots, y T_{d}-x_{d}\right)$ and $A=T_{\mathfrak{m} T}$ as in Lemma 3.1. Let $B$ be the integral closure of $A$. By hypothesis, $t=\operatorname{dim}_{k}(B / \operatorname{Jac}(B))$, where $\operatorname{Jac}(B)$ is the Jacobson radical of $B$. 
By Corollary 2.7 applied to $I A \subset A$, we have $e_{1}(I A) \leq\left(\begin{array}{c}e_{0}(I A)-n \\ 2\end{array}\right)$, where $n=$ $\left\lfloor\frac{\lambda(A / \overline{I A})-1}{t}\right\rfloor$. By Lemma 3.1, $e_{0}(I)=e_{0}(I A)$ and $e_{1}(I)=e_{1}(I A)$. Moreover, $\overline{I A}=\bar{I} A$ and hence $\lambda(R / \bar{I})=\lambda(A / \overline{I A})$. The conclusion follows.

Corollary 3.5. Let $(R, \mathfrak{m}, k)$ be an analytically unramified Cohen-Macaulay local domain with infinite residue field $k$. Let $I$ be an $\mathfrak{m}$-primary ideal of $R$, with integral closure $\bar{I}$. Let $t$ denote the essential rank of $I$. Suppose that $e_{1}(I)>\left(\begin{array}{c}e_{0}(I)-k \\ 2\end{array}\right)$ for some integer $k$. Then $t \geq \frac{\lambda(R / \bar{I})-1}{k}$.

Proof. By Theorem 3.4 we have $\left(\begin{array}{c}e_{0}(I)-k \\ 2\end{array}\right)<\left(\begin{array}{c}e_{0}(I)-n \\ 2\end{array}\right)$ with $n=\left\lfloor\frac{\lambda(R / \bar{I})-1}{t}\right\rfloor$. Hence $k>n$. So $k \geq \frac{\lambda(R / \bar{I})-1}{t}$.

Remark 3.6. Corollary 3.5 can be rephrased to give a lower bound on the essential rank of an $\mathfrak{m}$-primary ideal $I$ in an analytically unramified Cohen-Macaulay local domain $(R, \mathfrak{m})$ with algebraically closed residue field $k$. Namely, the condition that $e_{1}(I) \geq\left(\begin{array}{c}e_{0}(I)-k \\ 2\end{array}\right)$ in this corollary gives the smallest such value of $k$ as a root of a quadratic equation in $k$ with coefficients that are functions of $e_{0}(I), e_{1}(I)$. Solving this equation gives the following: if $t$ is the essential rank of $I$, then

$$
t \geq \frac{2(\lambda(R / \bar{I})-1)}{2 e_{0}(I)-1-\sqrt{8 e_{1}(I)+1}} .
$$

Note that, in general, $e_{0}(I)$ is larger than $\lambda(R / \bar{I})$, but we are subtracting $\sqrt{8 e_{1}(I)+1}$ in the denominator.

As mentioned in the introduction, Elias [E1] gives several bounds on $e_{1}(I)$. One of his bounds states that $e_{1}(I) \leq\left(\begin{array}{c}e_{0}(I)-k \\ 2\end{array}\right)$ if $I \subset \mathfrak{m}^{k}$ and the integral closure of $I$ is not the integral closure of $\mathfrak{m}^{k}$. The next corollary recovers this result and removes the condition that the integral closure of $I$ is not the integral closure of $\mathfrak{m}^{k}$.

Corollary 3.7. Let $(R, \mathfrak{m})$ be a Cohen-Macaulay local ring and let $I \subset R$ be an $\mathfrak{m}$-primary ideal. Suppose that $I \subset \mathfrak{m}^{k}$ for some $k \geq 2$. Then $e_{1}(I) \leq\left(\begin{array}{c}e_{0}(I)-k \\ 2\end{array}\right)$.

Proof. We proceed by induction on $d=\operatorname{dim}(R)$.

If $d=1$, the result follows from Theorem 2.1 provided that $\mathfrak{m}^{k}$ is not contained in the integral closure of $I$ as we have the sequence

$$
\mathfrak{m} \supset \overline{\mathfrak{m}^{2}} \supset \ldots \supset \overline{\mathfrak{m}^{k}} \supset \bar{I}
$$

in which every term is distinct.

Suppose that $\mathfrak{m}^{k} \subset \bar{I}$. Then $e_{0}(I)=k e_{0}(\mathfrak{m})$. Since $I^{n} \subset \mathfrak{m}^{n k}$, we see that

$$
e_{0}(\mathfrak{m})(n k)-e_{1}(\mathfrak{m}) \leq e_{0}(I) n-e_{1}(I)
$$

for all large $n$. Cancelling the first terms, it follows that $e_{1}(I) \leq e_{1}(\mathfrak{m})$. If $R$ is regular, then $e_{1}(\mathfrak{m})=0$, and therefore $e_{1}(I)=0$. Since $e_{0}(I)=k$ in this case, we are done by the convention that $\left(\begin{array}{l}0 \\ l\end{array}\right)=1$.

Henceforth we assume that $e_{0}(\mathfrak{m})>1$. By [KM] Cor. 3.3] we know that $e_{1}(\mathfrak{m}) \leq\left(\begin{array}{c}e_{0}(\mathfrak{m}) \\ 2\end{array}\right)$. Also note that $\left(\begin{array}{c}k e_{0}(\mathfrak{m})-k \\ 2\end{array}\right)=\left(\begin{array}{c}e_{0}(I)-k \\ 2\end{array}\right)$. But $\left(\begin{array}{c}e_{0}(\mathfrak{m}) \\ 2\end{array}\right) \leq\left(\begin{array}{c}k e_{0}(\mathfrak{m})-k \\ 2\end{array}\right)$ is equivalent to the inequality $e_{0}(\mathfrak{m}) \leq k^{2} e_{0}(\mathfrak{m})-k^{2}-k$, which holds since $k \geq 2$ and $e_{0}(\mathfrak{m}) \geq 2$.

Now let $d>1$. Let $a \in I$ be a superficial element for $I$. That is, there exists an integer $c \geq 0$ with $\left(I^{n+1}: a\right) \cap I^{c}=I^{n}$ for all $n \geq c$. Since $\operatorname{depth}(R)=d>0$ and $I$ is $\mathfrak{m}$-primary, $a$ is a nonzero divisor of $R$. 
Then $e_{0}(I)=e_{0}(I / a R)$ and $e_{1}(I)=e_{1}(I / a R)$. Further, $\operatorname{dim}(R / a R)=d-1$. Clearly, $I / a R \subset(\mathfrak{m} / a R)^{k}$. We are done by induction.

Discussion 3.8. In RV1] and RV2, the authors give other bounds on $e_{1}$. Notable among these is the bound [RV2, Theorem 3.2], which states that

$$
e_{1}(I) \leq\left(\begin{array}{c}
e_{0}(I) \\
2
\end{array}\right)-\left(\begin{array}{l}
b \\
2
\end{array}\right)-\lambda(R / I)+1,
$$

where $I$ is an $\mathfrak{m}$-primary ideal in a Cohen-Macaulay local ring of dimension $d$, and $b=\mu(I)-d$. See also E1 for different versions of this bound.

It is a little hard to compare our bound with the above bound in general. Just to give one example, consider the ring $R=k\left[\left[t^{7}, t^{8}, t^{9}, t^{10}\right]\right]$ and the ideal $I=$ $\left(t^{9}, t^{10}, t^{14}, t^{15}\right)$. Then $I$ is integrally closed since all the powers of $t$ in $R$ larger than 9 are in $I$. A few calculations show that $e_{0}(I)=e_{1}(I)=9, b=3$ and $\lambda(R / I)=3$. So our bound in Theorem 3.4 gives $e_{1}(I) \leq 21$, while the above bound is $e_{1}(I) \leq 31$. The above bound might be better when $I$ has many generators. In the example given, our bound is good simply because the essential rank is 1 .

\section{ACKNOWLEDGMENT}

We thank the referee for a careful reading of the paper and for providing new references.

Added after posting. The last sentence in the statement of Theorem 2.1 is not correct and should be disregarded. We thank Bill Heinzer for pointing this out to us.

\section{REFERENCES}

[ES] P. Eakin and A. Sathaye, Prestable ideals, J. Algebra 41 (1976), 439-454. MR0419428 $(54: 7449)$

[E1] J. Elias, On the first normalized Hilbert coefficient, J. Pure Applied Algebra 201 (2005), 116-125. MR2158750 (2006d:13017)

[E2] J. Elias, Upper bounds of Hilbert coefficients and Hilbert functions, Math. Proc. Cambridge Philos. Soc. 145 (2008), 87-94. MR2431640 (2009d:13020)

$[\mathrm{H}]$ C. Huneke, Complete ideals in two-dimensional regular local rings, Commutative Algebra, pp. 325-338, Math. Sci. Res. Inst. Publ., 15, Springer, New York, 1989. MR1015525 (90i:13020)

[I] S. Itoh, Coefficients of normal Hilbert polynomials, J. Algebra 150 (1992), 101-117. MR.1174891 (93j:13021)

[K] D. Katz, Note on multiplicity, Proc. Amer. Math. Soc. 104 (1988), 1021-1026. MR929434 (89d:13017)

[KM] D. Kirby and H. A. Mehran, A note on the coefficients of the Hilbert-Samuel polynomial for a Cohen-Macaulay module, J. London Math. Soc. (2) 25 (1982), 449-457. MR657501 (84a:13022)

[N] D. G. Northcott, A note on the coefficients of the abstract Hilbert function, J. London Math. Soc. 35 (1960), 209-214. MR0110731 (22:1599)

[Re] D. Rees, Degree functions in local rings, Proc. Cambridge Philos. Soc. 57 (1961), 1-7. MR0124353 (23:A1667)

[Ro] M.E. Rossi, A bound on the reduction number of a primary ideal, Proc. Amer. Math. Soc. 128 (2000), 1325-1332. MR 1670423 (2000j:13004)

[RV1] M.E. Rossi and G. Valla, Hilbert Functions of Filtered Modules, Lecture Notes of the Unione Matematica Italiana, 9, Springer, Heidelberg, 2010. MR 2723038

[RV2] M.E. Rossi and G. Valla, The Hilbert function of the Ratliff-Rush filtration, J. Pure Applied Algebra 201 (2005), 25-41. MR2158745 (2006g:13008) 
[RVV] M.E. Rossi, G. Valla, and W. Vasconcelos, Maximal Hilbert functions, Results Math. 39 (2001), 99-114. MR1817403 (2001m:13020)

[ST] V. Srinivas and V. Trivedi, On the Hilbert function of a Cohen-Macaulay local ring. J. Algebraic Geom. 6 (1997), 733-751. MR.1487234 (98i:13033)

$[\mathrm{SH}] \quad$ I. Swanson and C. Huneke, Integral closure of ideals, rings, and modules, London Mathematical Society Lecture Note Series, 336. Cambridge University Press, Cambridge, 2006. MR2266432(2008m:13013)

Department of Mathematics, University of Kansas, Lawrence, Kansas 66045

E-mail address: khanuma@math.ku.edu

Department of Mathematics, University of Kansas, Lawrence, Kansas 66045

E-mail address: huneke@math.ku.edu 